\title{
An analysis of angle, orientation, and location distortions in the bent line aftereffect*
}

\author{
ROGER B. HOWARD, STEVE R. MaCPEEK, and CHARLES BYRUM \\ Colgate University, Hamilton, New York 13346
}

\begin{abstract}
One hundred twenty-eight Ss evaluated the aftereffects of bent lines on four test figures. The results indicate that the $\mathrm{S}$ misperceives the orientation of the test figure rather than its angularity. There is a misperception of location, but it does not contribute to the basic aftereffect. These results, in conjunction with some recent findings in our laboratory, indicate that there are at least three components of the misperceptions occurring in most orientation studies.
\end{abstract}

In the bent line aftereffect, the objectively straight test $(\mathrm{T})$ line shown in Fig. 1.1 appears bent at the center to the left when preceded by an inspection (I) line bent to the right. The research reported here was designed to determine whether the bent line aftereffect is the result of angle, orientation, or location distortions. If the 180-deg angle formed by the $\mathrm{T}$ line is distorted (cf. Howard, 1971), then replacing the $T$ line with another $T$ figure which ends above the angle (Fig. 1.2) should eliminate the aftereffect. If the orientation of the upper and lower segments of the $\mathrm{T}$ line are distorted (cf. Gibson \& Radner, 1937), then the orientation of the $T$ line shown in Fig. 1.2 should also be affected. If the locations of the points composing the $\mathrm{T}$ lines are distorted (cf. Köhler \& Wallach, 1944), then the T segments shown in Figs. 1.3 and 1.4 should be displaced by the same amounts as the center and the ends, respectively, of the $\mathrm{T}$ line shown in Fig. 1.1.

\section{METHOD}

The Ss were 24 female and 104 male students from introductory courses in psychology. Forty Ss were used in each of the location conditions, 24 in the angle and 24 in the orientation conditions.

The stimuli used in the experiment and their dimensions are shown in Fig. 1. The bent line on the right of each configuration was the I figure and was always presented first. It was drawn in opaque black ink on a white card and shown in the second field of an Iconix three-field tachistoscope. The straight lines on the left in Figs. 1.1 and 1.2 and the short lines on the upper left in Figs. 1.3 and 1.4 were the $T$ figures. The short lines on the lower left were the comparison (C) figures used to determine the relative displacements of the $\mathrm{T}$ figures. Each $\mathrm{T}$ figure (and the corresponding $\mathrm{C}$ figure when appropriate) was drawn on a second card along with a fixation point at the same location as the point of the bent line and was shown in the third field of the tachistoscope. The remaining field, which was used as the foreand afterfield, contained a third white card with only a fixation point.

The aftereffect was determined by the method of recognition in which the S selected from one of four wall charts, the stimuli whose angularity, orientation, or relative location appeared most

*Preparation of this paper was supported in part by grants to the first author from the Colgate Research Council and the Sloan Foundation. It was completed while the author was a trainee at Duke University in the Sciences Related to the Nervous System. similar to the $\mathrm{T}$ or T-C combination presented on that trial (Fehrer \& Ganchrow, 1963). The charts for the angle, orientation, and the two location conditions showed lines whose distortion on the appropriate dimension successively increased and decreased in steps of 3 deg of visual angle.

The independent variables were the distance of the $\mathrm{T}$ figure from the center point of the I figure $(-30,-15,0,3,9,15,30$, 60 , and $90 \mathrm{~min}$ of visual angle), which we will call the interfigural distance, or IFD, and the nature of the T figures which defined the angle, orientation, upper location, and lower location conditions represented by Figs. 1.1-1.4, respectively.

Each S participated in only one of the four conditions. A session consisted of two blocks of 18 trials, 9 experimental trials defined by the nine IFDs and 9 control trials defined by replacing the I figure in each experimental configuration by a fixation dot. The 18 trials were presented in random order in each block. The first block was preceded by 6 practice trials selected randomly from the 18 experimental and control combinations and followed by a 2-min rest. The second block was preceded by 2 randomly selected practice trials. The I and T figures were presented for 200 and $100 \mathrm{msec}$, respectively, with no pause between them. The luminance from each card was $10 \mathrm{fc}$, and all viewing was monocular with the S's dominant eye.
.1.

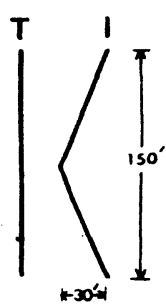

1.3

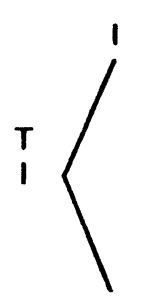

C
12

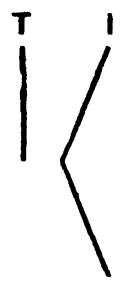

14

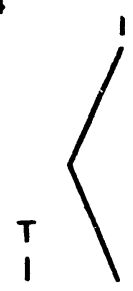

C
Fig. 1. Stimulus configurations used in the angle (1.1), orientation (1.2), upper location (1.3), and lower location (1.4) conditions. $I=$ inspection, $T=$ test, $C=$ comparison figure. 


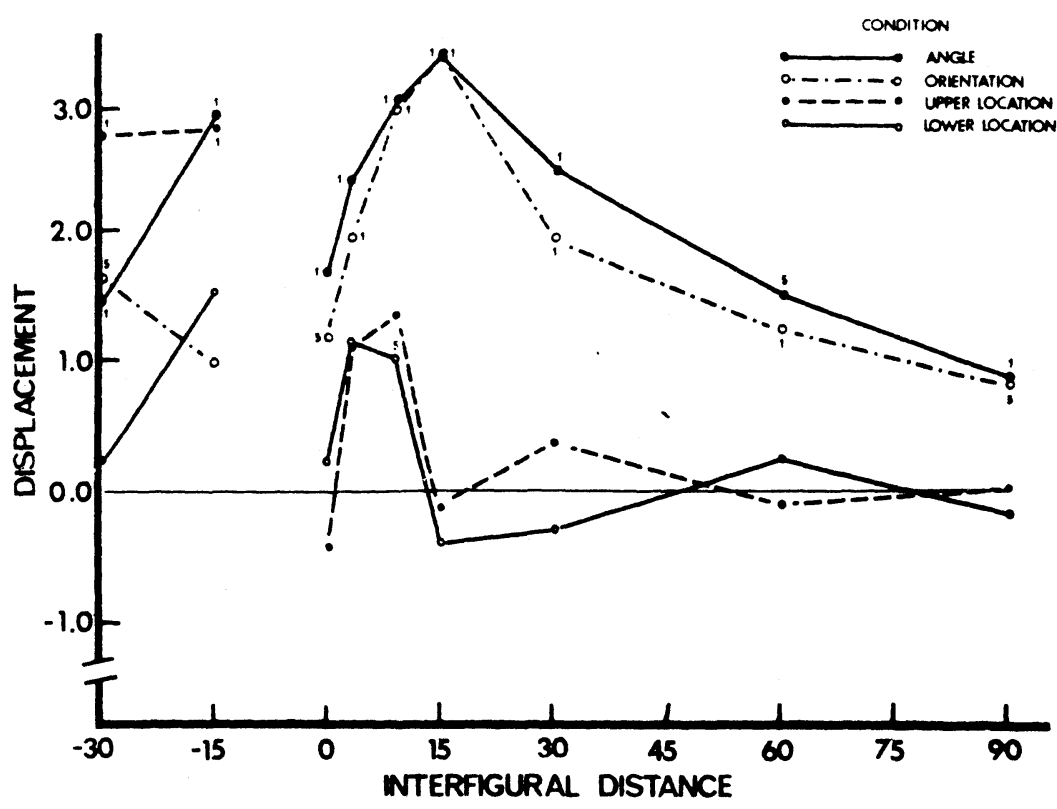

Fig. 2. Misperceptions found in the four experimental conditions plotted as functions of the interfigural distance (IFD), both in minutes of visual angle.

\section{RESULTS AND DISCUSSION}

Figure 2 shows the misperception scores, obtained by subtracting the control from the experimental judgments, as functions of the IFD. Hartley F-maximum tests (Winer, 1971) were performed on the data from each condition. None of the tests was significant. T tests were therefore computed between the mean at each of the 36 data points and a null value of zero misperceptions. A " 5 " or a " 1 " at a point in Fig. 2 indicates that the difference is significant with $a=0.05$ or 0.01 , respectively.

The data suggest that the bent line I figure affects the orientation of the $\mathrm{T}$ figure, but has no detectable effect on the angularity of the $\mathrm{T}$ figure per se. If the I figure has its influence on the angularity of the $T$ figure, then only the angle condition should show the traditional inverted-U function relating magnitude of misperception to IFD. In fact, the angle and orientation conditions produce very similar functions, suggesting that it is the orientation of the two halves of the I line which contribute to the misperception rather than the angle which they form.

The data also suggest that there is a location effect which does not contribute to the misperception found in the orientation and angle conditions. In order for location misperceptions to simulate angle or orientation misperceptions, the part of the line closest to the end points of the I figure must be displaced more than the part closest to its center. Thus the $\mathrm{T}$ figure in the lower location condition shown in Fig. 1.4 should be displaced considerably more than the $\mathrm{T}$ figure in the upper location condition shown in Fig. 1.3. Figure 2 shows that both configurations produce misperceptions which are very similar functions of the IFD. Hence the orientation misperception found in the orientation and angle conditions is not produced by location misperception.
Further consideration of the location effect suggests that it has several unorthodox properties. The traditional function relating location misperceptions to the separation between the I and $\mathrm{T}$ contours shows a peak at about $9 \mathrm{~min}$ and rapid decay to around zero misperception by 15-30 min (Köhler \& Wallach, 1944; cf. Howard, 1971). This is the pattern found here with IFD as the independent variable. Note, however, that IFD is measured relative to the point of the I figure. IFD for the upper location condition is, therefore, roughly equivalent to the intercontour distance used with traditional location studies. This is not true for the lower location condition where an additional 26 min of visual angle must be added to the IFD to find the true separation. Hence the similar functions are found despite a difference in the contour separation greater than the range of the entire location misperception found in the experiment. The location detectors must be tuned to a width of about $15 \mathrm{~min}$ yet produce the same functions despite a constant difference of $26 \mathrm{~min}$.

The results of this experiment, taken in conjunction with the data from several other recent experiments, suggest an unparsimonious view of visual misperceptions. Our data indicate that a bent line produces misperceptions of orientation and location. Morant and Harris (1965) and Howard, Kennedy, and Boxer (1974) have shown that there are at least two components to the orientation misperception produced by figures which would be identical to those used in our orientation condition if the lower half of the bent line were removed. One component is similar to the orientation misperception suggested by Gibson and Radner (1937) and might be equated with the orientation misperception found here. The second component is similar to the location component suggested by Köhler and Wallach. It differs from our location effect in that it is not localized, and it must affect the parts of the $T$ line unequally because it produces changes in the orientation 
of the $\mathrm{T}$ figure. Our data suggest that if a location misperception does exist, its peak occurs near an IFD of $9 \mathrm{~min}$ and drops to zero by $15 \mathrm{~min}$. Morant and Harris (1965) and Howard, Kennedy, and Bower (1974), however, have shown that the effect is present at distances up to $6 \mathrm{deg}$. Although there are sufficient differences in the designs to make absolute conclusions hazardous, it seems likely that there are at least three misperceptions involved in the two studies. Thus, the original hope of many theorists that misperceptions are produced by a few mechanisms operating on sets of neurons sensitive to specific attributes is incorrect.

\section{REFERENCES}

Fehrer, E., \& Ganchrow, D. Effects of exposure variables on figural aftereffects under tachistoscopic presentation. Journal of Experimental Psychology, 1963, 66, 506-513.

Gibson, J. J., \& R adner, M. Adaptation, after-effect and contrast in the perception of tilted lines. 1. Quantitative studies. Journal of Experimental Psychology, 1937, 20, 453-467.

Howard, R. B. Neurophy siological models of figural aftereffects and visual illusions. Psychonomic Monograph Supplements, 1971, 4, 57-72.

Howard, R. B., Kennedy, A, \& Boxer, P A mathematical analysis of figural aftereffects and normalization components of tilt aftereffects. Bulletin of the Psychonomic Society, 1974 , in press.

Köhler, W., \& Wallach, H. Figural after-effects: An investigation of visual processes. Proceedings of the American Philosophical Society, 1944, 88, 269-357.

Morant, R. B., \& Harris, J. R. Two different after-effects of exposure to visual tilts. American Journal of Psychology, 1965, 78, 218-226.

Winer, B. J. Statistical principles in experimental design. (2nd ed.) New York: McGraw-Hill, 1971.

(Received for publication May 31, 1974.)

\section{ERRATUM}

WITTE, K. L., \& HUNTERMARK, J. The effects of instructions, evaluative feedback, and knowledge of results upon the short-term retention of ninth graders. Bulletin of the Psychonomic Society, 1975, 5, 79-81. Column 2, page 80, top line should read as follows: "The Instructions by Feedback by Knowledge of Results Interaction was significant $[\mathrm{F}=5.28, \mathrm{df}=1 / 32]$." 